\title{
Unruhige Gewässer
}

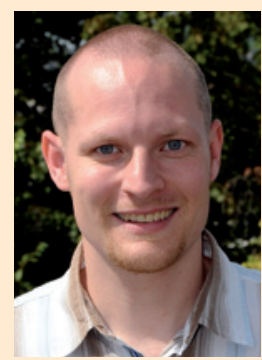

Christian Schäfer, Stuttgart
Seit Sommer 2012 waren es stürmische Zeiten für alle Organisationen und Menschen, die direkt mit der Organspende zu tun haben - also u.a. für Transplantationsmediziner, -pflegekräfte, die Deutsche Stiftung Organtransplantation (DSO), Eurotransplant (ET) und natürlich für die Patienten, die dringend ein neues Organ benötigen. All jene waren davon besonders betroffen, als die Skandale zu Unregelmäßigkeiten bei der Organvergabe wie unheilvolle Wellen über Deutschland rollten. Zum Glück waren relativ wenige Kliniken hierin verwickelt. Aber auch diese wenigen Fälle haben ausgereicht, um das Vertrauen der Bevölkerung in das System nachhaltig zu erschüttern.

Negativ wirkt hier u.a. auch der immer noch andauernde Göttinger Transplantationsprozess mit entsprechenden Schlagzeilen, die in unregelmäßigen Abständen auf die Bürger einprasseln. Dies hat anscheinend einen lang anhaltenden Effekt auf die Bereitschaft, seine Organe nach dem Hirntod zu spenden (dokumentiert über einen Organspendeausweis): Der DSO-Jahresbericht „Organspende und Transplantation in Deutschland 2013“ zeigt u.a., dass die Zahl der Organspenden 2013 im Vergleich zu 2012 um 16,3\% gesunken ist.

Außerdem hat die sich schon lange hinziehende Debatte um die korrekte Feststellung des Hirntodes sicherlich auch nicht gerade dabei geholfen, Vertrauen zurückzugewinnen. Zugespitzt gesagt ist die Frage, die sich wohl viele stellen, folgende: Wer will schon gerne festlegen, dass man seine Organe nach dem Tod spendet, wenn man das Gefühl bekommt, dass nicht einmal Ärzte sich sicher sind, wann ein Mensch wirklich tot ist und wie man das am besten feststellt? Das Problem ist bei diesem hochkomplexen Thema derzeit, dass bei der geplanten neuen Hirntodrichtlinie der Bundesärztekammer (BÄK) alle Expertenmeinungen und auch neueste wissenschaftliche Erkenntnisse einfließen - dies ist sorgsam unter einen Hut zu bringen. Daher hat sich der Vorsitzende des Wissenschaftlichen Beirats der BÄK Prof. Peter C. Scriba, München, gegen Andeutungen gewehrt, die Ausarbeitung einer Empfehlung für die neue Richtlinie ginge zu lange. Diese Vorwürfe hatte der ehemalige Interimsvorstand der DSO Dr. Rainer Hess, Frankfurt am Main, Ende März kurz vor seinem Ausscheiden aus dem Amt gegenüber dem Nachrichtenmagazin „Der Spiegel“ in einem Interview indirekt formuliert. Immerhin kündigte Scriba gegenüber der „Ärzte Zeitung“ an, dass es möglicherweise noch dieses Jahr zur Richtliniennovelle kommen könnte.

Was bleibt als Fazit? Wohl dies: Es ist notwendig, das bedrohlich in die Schieflage geratene Schiff mit dem Namen „Organspende in Deutschland“ aus den unruhigen Gewässern herauszusteuern. Hierbei haben der seit dem 1. April neue Medizinische Vorstand der DSO Dr. Axel Rahmel und seine Kollegen sicherlich noch einiges vor sich. Mit Rahmel - der ehemalige medizinische Direktor von ET - ist hier glücklicherweise ein würdiger Nachfolger für Hess gefunden, der das Potenzial hat, die verdienstvolle Arbeit seines Vorgängers sehr gut weiterzuführen. Sicherlich kann auch der „Tag der Organspende“ unter dem Motto „Richtig. Wichtig. Lebenswichtig." dabei helfen, um die Bevölkerung behutsam an das sensible Thema heranzuführen. Und wer weiß, vielleicht entscheidet sich am 7. Juni (dem Veranstaltungsdatum) der eine oder andere für ein „Ja“ in seinem Organspendeausweis.

Auch in der aktuellen Ausgabe der Dialyse aktuell wollen wir mit dem Schwerpunkt „Nierentransplantation“ ab Seite 184 auf dieses wichtige und spannende Thema aufmerksam machen. So stellen die kompetenten Autoren u. a. die Meilensteine in der Geschichte der Nierentransplantation vor und beschäftigen sich mit den Besonderheiten der Langzeitimmunsuppression. Und natürlich können Ärzte in dieser Ausgabe mittels des Fragebogens bei mindestens 70\% richtig beantworteten Fragen wieder 3 CME-Punkte sammeln. Ich wünsche Ihnen hierbei viel Erfolg und eine angenehme Lektüre! 